\title{
An enzymatically fortified ginseng extract inhibits proliferation and induces apoptosis of KATO3 human gastric cancer cells via modulation of Bax, mTOR, PKB and IKB $\alpha$
}

\author{
JEONG-WON HWANG $^{1 *}$, YOUNG-MI BAEK $^{2 *}$, IK-SOON JANG $^{1 *}$, KYEONG EUN YANG $^{1}$, DONG-GI LEE ${ }^{1}$, \\ SO-JUNG YOON ${ }^{3}$, JAERANG RHO $^{3}, \mathrm{CHONG}^{-K W A N ~ C H O}{ }^{2}$, YEON-WEOL LEE ${ }^{2}, \mathrm{KI}^{-R O K} \mathrm{KWON}^{4}$, \\ HWA-SEUNG YOO ${ }^{2}$, JUNG-SUK SUNG ${ }^{5}$, SHIN KIM ${ }^{6}$, JONG-WOOK PARK ${ }^{6}$, \\ BYEONG-CHURL JANG ${ }^{7}$ and JONG-SOON CHOI ${ }^{1,3}$
}

\author{
${ }^{1}$ Division of Life Science, Korea Basic Science Institute, Daejeon 305-333; ${ }^{2}$ East-West Cancer Center, Daejeon University, \\ Daejeon 302-120; ${ }^{3}$ Graduate School of Analytical Science and Technology, Chungnam National University, Daejeon 305-764; \\ ${ }^{4}$ Research Center of Pharmacopucture Medicine, Korean Pharmacopuncture Institute, Seoul 157-200; \\ ${ }^{5}$ Department of Life Science, Dongguk University Seoul, Seoul 100-715; \\ Departments of ${ }^{6}$ Immunology and ${ }^{7}$ Molecular Medicine, College of Medicine, \\ Keimyung University, Daegu 704-701, Republic of Korea
}

Received November 11, 2013; Accepted June 17, 2014

DOI: $10.3892 / \mathrm{mmr} .2014 .2704$

\begin{abstract}
Accumulative evidence suggests ginseng extract and/or its major components, ginsenosides and compound $\mathrm{K}$, a metabolized ginseng saponin, have anti-cancer effects. In the present study, the effects of a ginseng butanolic extract (GBX) and an enzymatically fortified ginseng extract (FGX), with enriched ginsenosides and compound $\mathrm{K}$, on the growth of KATO3 human gastric cancer cells were investigated using a cell viability assay. While treatment with GBX at 31.25-125 mg/ml for $24 \mathrm{~h}$ did not affect the proliferation of KATO3 cells, FGX under the same conditions inhibited cell proliferation in a concentration-dependent manner. Furthermore, Annexin V/PI-staining and flow cytometric analysis demonstrated that the population of apoptotic KATO3 cells was increased following treatment with FGX, which was greater than in the GBX-treated cells, suggesting that FGX had a stronger apoptotic effect than GBX. To investigate
\end{abstract}

Correspondence to: Professor Byeong-Churl Jang, Department of Molecular Medicine, College of Medicine, Keimyung University, 1095 Dalgubeol-daero, Dalseo-gu, Daegu 704-701, Republic of Korea

E-mail: jangbc12@kmu.ac.kr

Dr Jong-Soon Choi, Division of Life Science, Korea Basic Science Institute, 169-148 Gwahangno, Yuseong-gu, Daejeon 305-333, Republic of Korea

E-mail: jschoi@kbsi.re.kr

*Contributed equally

Key words: fortified ginseng extract, $\mathrm{Bax}, \mathrm{I} \kappa \mathrm{B} \alpha, \mathrm{mTOR}, \mathrm{PKB}$, KATO3 cells the underlying mechanism of the cytostatic and cytotoxic effects of the ginseng extracts, apoptosis-associated proteins were assessed using western blot analysis. The data revealed higher expression levels of B-cell lymphoma 2-associated X protein (Bax), lower expression of nuclear factor of kappa light polypeptide gene enhancer in B-cells inhibitor $\alpha$ $(\mathrm{I} \kappa \mathrm{B} \alpha$ ) and reduced phosphorylation of mammalian target of rapamycin (mTOR) and protein kinase $B(P K B)$ in the FGX-treated KATO3 cells than in the GBX-treated cells. Collectively, these results demonstrated for the first time, to the best of our knowledge, that FGX had stronger anti-proliferative and pro-apoptotic effects on KATO3 cells than GBX. The anti-proliferative and/or pro-apoptotic effects of FGX appeared to be mediated via the upregulation of Bax, IкB $\alpha$ proteolysis (activation of nuclear factor $-\kappa \mathrm{B}$ ) and the blocking of mTOR and PKB signals.

\section{Introduction}

Ginseng has been widely used as a herbal medicine in Eastern Asian countries, including Korea. Ginseng is a species of perennial plant belonging to the genus Panax $(P)$ in the family Araliaceae. Ginseng species are found only in the Northern hemisphere from Siberia to Vietnam. Among the ginseng species, Asian ginseng ( $P$. ginseng) and American ginseng (P. quinquefolius) are the best characterized. Previously, Asafu-Adjaye and Wong (1) have demonstrated the quantitative and qualitative profiling of ginsenosides (ginseng saponins) in dry root powder from $P$. ginseng and $P$. quinquefolius, respectively. Evidence strongly suggests that $P$. ginseng contains $>30$ pharmacologically active saponins, including Rh1, Rh2, $\operatorname{Rg} 3$ and $\operatorname{Rg} 5$ (2). There is, of further importance, evidence strongly supporting that ginseng extract and/or ginsenosides (Rg3, Rh2, Rb2, compound K and F2) have anti-carcinogenic 
activities against numerous types of cancer, including colon, liver and breast cancer (3-6). In addition, the preventive activities of ginseng saponins by inhibiting cancer cell growth and metastasis in vivo and in vitro have also been previously proposed (7). In a recent study, it was demonstrated that butanolic extract of mountain ginseng had anti-proliferative and pro-apoptotic effects on A549 human lung cancer cells via modulation of the expression and/or activity of p53, caspase-3 and nuclear factor (NF)- $\mathrm{B}$ (8). Furthermore, a previous study by our group provided evidence that Rb1, Rb2 and Rg1, three of the most abundant ginsenosides in the ginseng extract, induced apoptosis in A549 cells through extrinsic (death receptor-mediated) apoptotic pathways (9). In addition, there is clinical evidence that red ginseng powder effectively enhances postoperative immunity and survival in patients with stage III gastric cancer (10), suggesting the potential clinical applications of ginseng preparations against gastric cancer.

Gastric cancer is the 4th most frequent cancer and the second leading cause of cancer-associated mortality worldwide. Although $50 \%$ of gastric tumors are detected at an early stage in asymptomatic individuals, often when symptoms occur, the cancer has already reached advanced stages and may proceed to metastasis, leading to a poor prognosis for patients (11). At present, the current treatment strategies for gastric cancer include surgery, chemotherapy and/or radiotherapy (12). Among these, radical surgery offers the only chance of cure for patients with operable gastric cancer, but the outcomes remain generally poor due to a high rate of relapse post gastric surgery. Therefore, multimodality therapy using chemotherapy, radiation or a combination of both has been evaluated in different parts of the world to improve the outcomes from surgery alone $(12,13)$. Numerous chemotherapeutic drugs, including 5-fluorouracil, doxorubicin, mitomycin $\mathrm{C}$, cisplatin and taxotere, have been used individually or in combination for the treatment of gastric cancer (14). Furthermore, there is evidence that everolimus, an inhibitor of the mammalian target of rapamycin (mTOR) pathway, is also effective and well tolerated in phase I/II studies of patients with chemotherapy-refractory metastatic gastric cancer (15). However, due to the poor long-term outcomes associated with current chemotherapy treatment of patients with advanced gastric cancer, there is still a need for the development of novel targeted agents that may confer an improved survival benefit.

In the present study, the anti-cancer effect and mechanism of a fortified ginseng extract (FGX), newly prepared from $P$. ginseng root butanolic extract (GBX) by the treatment of two enzymes, laminarinase (polysaccharides-degrading enzyme) and pectinase (pectin-degrading enzyme), in KATO3 human gastric cancer cells were investigated.

\section{Materials and methods}

Materials. Dulbecco's modified Eagle's medium (DMEM) was purchased from Sigma-Aldrich (St. Louis, MO, USA). The 96-well plates used were obtained from NUNC (Roskilde, Denmark). Fetal bovine serum (FBS), phosphate-buffered saline (PBS), penicillin-streptomycin and L-glutamate were obtained from Gibco-BRL (Carlsbad, CA, USA). The Annexin-V-carboxyfluorescein (FLUOS) staining kit was provided from Roche Diagnostics GmbH (Mannheim,
Germany). An antibody against nuclear factor of kappa light polypeptide gene enhancer in B-cells inhibitor $\alpha(\mathrm{I} \kappa \mathrm{B} \alpha)$ was purchased from Santa Cruz Biotechnology, Inc. (Santa Cruz, CA, USA). B-cell lymphoma-2-associated X (Bax) and B-cell lymphoma-2 (Bcl-2) antibodies were obtained from Abcam (Cambridge, MA, USA). Antibodies against protein kinase B (PKB), phosphorylated (p)-PKB, mTOR and p-mTOR were purchased from Cell Signaling Technology, Inc. (Beverly, MA, USA). Secondary antibodies of horseradish peroxidase (HRP)-conjugated anti-rabbit immunoglobulin (Ig) $\mathrm{G}$ were purchased from Invitrogen Life Technologies, Inc. (Carlsbad, CA, USA). Other reagents, including mouse monoclonal anti-human actin antibody, were purchased from Sigma-Aldrich.

Cell culture. KATO3, a human gastric cancer cell line, was obtained from American Type Culture Collection (Rockville, MD, USA). KATO3 cells were grown in DMEM supplemented with $10 \%(\mathrm{v} / \mathrm{v})$ FBS and $1 \%(\mathrm{w} / \mathrm{v})$ penicillin-streptomycin in a cell culture chamber at $37^{\circ} \mathrm{C}$ with $5 \%(\mathrm{v} / \mathrm{v}) \mathrm{CO}_{2}$.

Preparation of an enzymatically FGX from GBX. The root of regular ginseng (four years old) was purchased from National Agricultural Cooperative Foundation (Chuncheongnam-do, Korea). A total of $20 \mathrm{~g}$ pulverized ginseng root powder was suspended in $380 \mathrm{ml}$ distilled water and then sterilized at $121^{\circ} \mathrm{C}$ for $15 \mathrm{~min}$. To reduce the complexity of components in cultivated regular ginseng root, the extract was fractionated via extraction with various solvents, including water, methanol and butanol. Among them, ginseng butanolic extract (GBX) contained compounds with specific anti-gastric cancer activity. GBX was used as a control of regular ginseng. In addition, the suspension was treated with an aliquot of filter-sterilized commercial enzymes (laminarinase, pectinase) at an equimolar ratio (1:1, specific activity units), and the mixture was then incubated at $40^{\circ} \mathrm{C}$ for two days and evaporated to dryness at $60^{\circ} \mathrm{C}$. These enzyme-modified ginseng powders were suspended in $400 \mathrm{ml} \mathrm{80 \%} \mathrm{(v/v)} \mathrm{methanol.} \mathrm{The} \mathrm{suspension} \mathrm{was} \mathrm{treated} \mathrm{in} \mathrm{an}$ ultrasound bath for $5 \mathrm{~min}$ and filtered through Whatman no. 2 filter paper (Whatman International Ltd., Maidstone, UK). The wet powder on the filter paper was collected, suspended, treated in an ultrasound bath and filtered in the same manner once again. The two filtrates were combined and evaporated to dryness at $50^{\circ} \mathrm{C}$. The methanolic extract was dissolved in $200 \mathrm{ml}$ of distilled water and washed with $200 \mathrm{ml}$ ethyl acetate in a separation funnel and then extracted with $200 \mathrm{ml}$ butanol. The butanolic extract was evaporated to dryness at $50^{\circ} \mathrm{C}$ and redissolved to the concentration of $10 \%$ (w/v) in $70 \%$ ethanol. This final extract was termed as an enzymatically FGX herein.

High-performance liquid chromatography (HPLC) analysis of the ginsenoside profiles in FGX and GBX. Standard ginsenosides, including Rg1, Re, Rf, Rh1, Rb1, Rc, Rb2, f1, Rd, Rg3(S), Rg3(R), PPT, compound K, Rh2 and PPD, were obtained from ChromaDex, Inc. (Irvine, CA, USA) and the profiles were analyzed using an Acquity ultra (U)PLC system (Waters, Milford, MA, USA) with an Acquity BEH $\mathrm{C}_{18}$ HPLC column. The mobile phase consisted of solution $\mathrm{A}\left(\mathrm{CH}_{3} \mathrm{CN}\right.$, HPLC-grade; JT Baker, Center Valley, PA, USA) and solution B (Milli-Q $\mathrm{H}_{2} \mathrm{O}$; Millipore, Billerica, MA, USA). The flow 
A

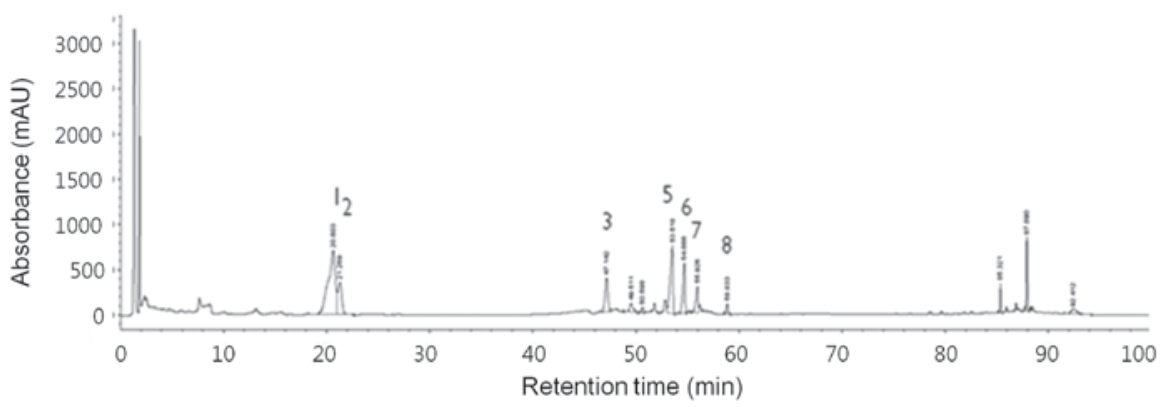

B

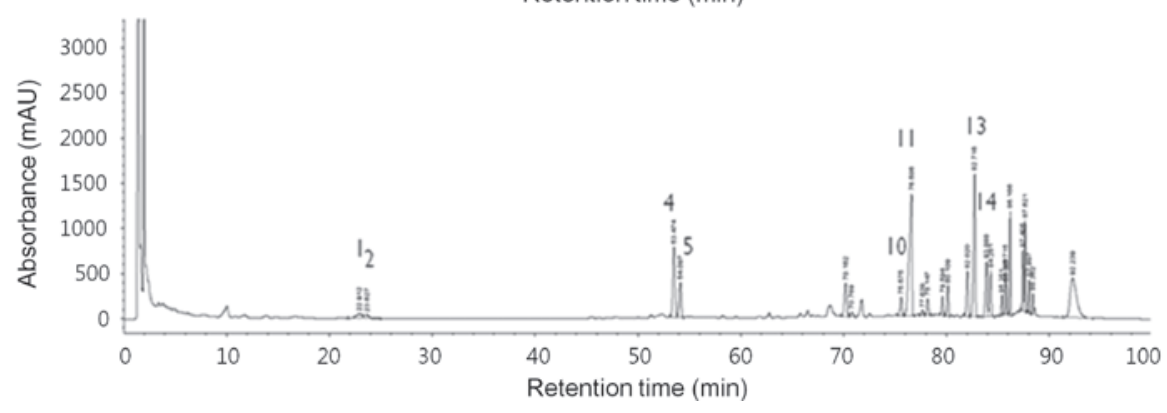

Figure 1. High-performance liquid ultraviolet chromatograms of ginseng root extracts. (A) Ginseng butanol extract and (B) enzymatically fortified ginseng extract of roots treated with laminarinase and pectinase.

rate was set at $0.6 \mathrm{ml} / \mathrm{min}$ and the injection of volume was fixed at $2 \mu \mathrm{l}$. The separation of ginsenosides was performed using an isocratic gradient of $100 \%$ solution A for $27 \mathrm{~min}$. The column temperature was maintained at room temperature during the separation and the ultraviolet (UV) diode array detection was set at $203 \mathrm{~nm}$. For the confirmation of a reliable retention time of sample ginsenoside, the individual ginsenoside was identified and quantified by comparing it with the retention times of the standard ginsenosides.

Cell viability assay. KATO3 cells $\left(5 \times 10^{3}\right.$ cells/well) were seeded in a 96-well plate overnight. The cells were then treated with GBX or FGX at various concentrations for $24 \mathrm{~h}$, followed by a measurement of cell viability using the Cell Counting kit-8 (Dojindo Molecular Technologies, Inc., Kumamoto, Japan ), as described previously (8). The cell viability was determined by measuring the absorbance at $450 \mathrm{~nm}$ using a microplate reader (Sunrise ${ }^{\mathrm{TM}}$-Basic; Tecan, Mannedorf, Switzerland). Each data point was determined by triplicate experiments.

Identification of apoptosis by PI-Annexin-V staining. The cell apoptosis was assessed using an Annexin-V-FLUOS Staining kit (Roche Diagnostics), according to the manufacturer's instructions. KATO3 cells were incubated with GBX or FGX for $24 \mathrm{~h}$, scraped and washed twice with PBS, and centrifuged at $930 \mathrm{xg}$ for $5 \mathrm{~min}$ at $4^{\circ} \mathrm{C}$. The cells were then incubated with $0.2 \mathrm{mg} / \mathrm{ml}$ Annexin-V-FLUOS and $1.4 \mathrm{mg} / \mathrm{ml}$ DNA stain propidium iodide (PI) for $15 \mathrm{~min}$ at room temperature. The measurements were conducted on a FACSCalibur flow cytometer (Becton Dickinson Immunocytometry Systems, San Jose, CA, USA) at $488 \mathrm{~nm}$ excitation and $530 / 30 \mathrm{~nm}$ band pass filter for fluorescence detection, and $670 \mathrm{~nm}$ high pass filter for PI detection. The data were analyzed by WinMDI V2.9 software (Joe Trotter, The Scripps Institute, La Jolla, CA, USA).
Western blot analysis. The expression levels of intracellular proteins from GBX- and FBX-treated cells were examined by western blotting, as described previously (8). The denatured protein of $30 \mu \mathrm{g}$ was separated by $12 \%$ SDS-PAGE and then transferred onto a nitrocellulose membrane. The transferred nitrocellulose membrane was stained with Ponceau $\mathrm{S}$ (Sigma-Aldrich) to visualize the proteins. The blotted membrane was blocked for $1 \mathrm{~h}$ with $5 \%$ (w/v) skimmed milk in TTBS [0.1\% (v/v) Tween-20 and Tris-buffered saline], followed by incubation with the respective primary antibody for $\mathrm{I} \kappa \mathrm{B} \alpha(1: 1,000)$, Bcl-2 (1:1,000), Bax (1:2,000), PKB (1:1,000), p-PKB (1:1,000), mTOR $(1: 2,000)$, p-mTOR $(1: 1,000)$ and actin $(1: 5,000)$ at room temperature (RT) for $2 \mathrm{~h}$ or at $4^{\circ} \mathrm{C}$ overnight. The membrane was washed three times for $5 \mathrm{~min}$ with TTBS and further incubated with secondary antibody of HRP-conjugated rabbit anti-goat IgG $(1: 2,000)$ in TTBS containing 5\% (w/v) skimmed milk at RT for $1 \mathrm{~h}$. The membrane was then rinsed three times with TTBS-0.1\% (v/v) Tween-20 for $5 \mathrm{~min}$. Then, the Pierce enhanced chemiluminescence system (Thermo Scientific, San Jose, CA, USA) was used to develop the proteins on an X-ray film (Kodak, Seoul, Korea). The expression levels of each protein were quantitatively analyzed, with those of actin protein used as an internal control.

Statistical analyses. The GraphPad Prism version 5.0 software (GraphPad, San Diego, CA, USA) was used for statistical analysis. A Student's t-test was used to assess the difference between the GBX and FGX-treated group. The $\mathrm{IC}_{50}$ values were calculated by non-linear curve fit at four data points and presented as the mean \pm standard deviation. $\mathrm{P}<0.001$ was considered to indicate a statistically significant difference.

\section{Results}

Ginsenoside profiles in FGX and GBX. Primarily, HPLC-UV analysis was conducted to generate a quantitative profile of the 
Table I. Quantities of ginsenosides in GBX and FGX.

\begin{tabular}{ccccccccccccccc}
\hline & \multicolumn{110}{c}{ Guantity of Ginsenoside (mg/g) } \\
\cline { 2 - 14 } & Rg1 & Re & Rf & Rh1 & Rb1 & Rc & Rb2 & F1 & Rd & Rg3(S) & Rg3(S)R & PPT & ComK & Rh2 \\
\hline GBX & 7.00 & 7.21 & 2.54 & - & 7.65 & 4.68 & 2.32 & 2.02 & - & - & - & - & - & - \\
FGX & 0.61 & 1.02 & - & 2.74 & 2.65 & - & - & - & - & 0.94 & 8.58 & - & 4.73 & 2.65 \\
\hline
\end{tabular}

GBX, ginseng butanol extract; FGX, fortified ginseng extract; ComK, compound K.

A

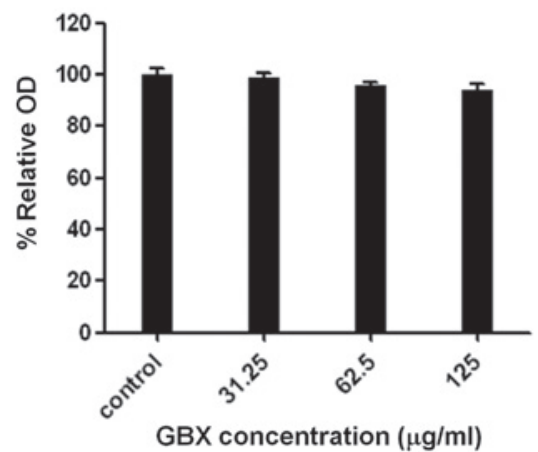

B
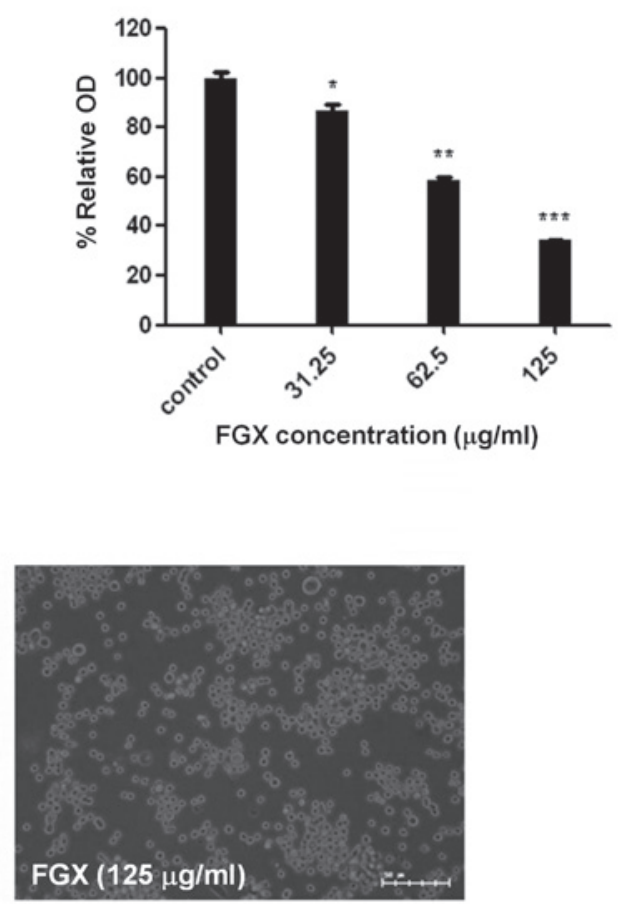

Figure 2. Effect of GBX or FGX on growth of KATO3 cells. (A) KATO3 cells were treated without or with the indicated concentrations of GBX or FGX for 24 h. Bar graph are presented as the mean \pm standard deviation from triplicate experiments. ${ }^{*} \mathrm{P}<0.05,{ }^{* *} \mathrm{P}<0.01$ and ${ }^{* * *} \mathrm{P}<0.001$ vs. control. (B) Light microscopy images of KATO3 cells administered with GBX $(125 \mu \mathrm{g} / \mathrm{ml})$ and FGX (125 $\mu \mathrm{g} / \mathrm{ml})$ for $24 \mathrm{~h}$, respectively (magnification, $\mathrm{x} 400)$. GBX, ginseng butanol extract; FGX, fortified ginseng extract; OD, optical density.

ginsenoside composition of FGX and GBX. As demonstrated in Fig. 1, the prominent ginsenoside peaks were present in the chromatograms of FGX and GBX. A total of seven ginsenosides, including $\mathrm{Rg} 1, \mathrm{Re}, \mathrm{Rf}, \mathrm{Rb} 1, \mathrm{Rc}, \mathrm{Rb} 2$ and $\mathrm{F} 1$, were presented in GBX. By contrast, five ginsenosides, including Rh1, Rg3(S), Rg3(R), compound K and Rh2, were exclusively detected in FGX. In particular, in FGX, the most intensive HPLC peak was $\operatorname{Rg} 3(\mathrm{R})$ in the range of 75-80 min. Therefore, considering that $\mathrm{Rg} 3, \mathrm{Rh} 2$ and compound $\mathrm{K}$ are major ginsenosides with anti-cancer activity, these results suggested the enrichment of more ginsenosides with anti-cancer activity in FGX compared with the ginsenosides in GBX. The quantitative data of the ginsenoside profiles in FGX and GBX are listed in detail in Table I.

FGX treatment induces a marked loss of KATO3 cells. Next, the effects of FGX and GBX on the proliferation and the morphology of KATO3 human gastric cancer cells was examined. As demonstrated in Fig. 2A, FGX inhibited the growth of KATO3 in a dose-dependent manner, while GBX exhibited no clear inhibition at up to $125 \mu \mathrm{g} / \mathrm{ml}$. Furthermore, KATO3 cells had round shapes but there was no marked difference in the morphology of KATO3 cells treated with FGX and GBX at $125 \mu \mathrm{g} / \mathrm{ml}$ for $24 \mathrm{~h}$, respectively, as revealed in Fig. $2 \mathrm{~B}$. However, the number of KATO3 cells following treatment with FGX (125 $\mu \mathrm{g} / \mathrm{ml}, 24 \mathrm{~h})$ was significantly lower compared with that following treatment with GBX $(125 \mu \mathrm{g} / \mathrm{ml}, 24 \mathrm{~h})$ under these experimental conditions.

FGX treatment induces apoptosis of KATO3 cells. It was further investigated whether FGX induces apoptosis of KATO3 cells by using Annexin V/PI staining-based flow cytometric analysis, in which cells in early stages of apoptosis (Annexin-stained, non-disrupted cells) were distinguished from those necrotic cells (disrupted or lysed cells). The quantitative data of the flow cytometric analysis, as revealed in Fig. 3, demonstrated that treatment with FGX and GBX at $125 \mu \mathrm{g} / \mathrm{ml}$ for $24 \mathrm{~h}$ resulted in $\sim 12.94$ and $5.04 \%$ cells at early 

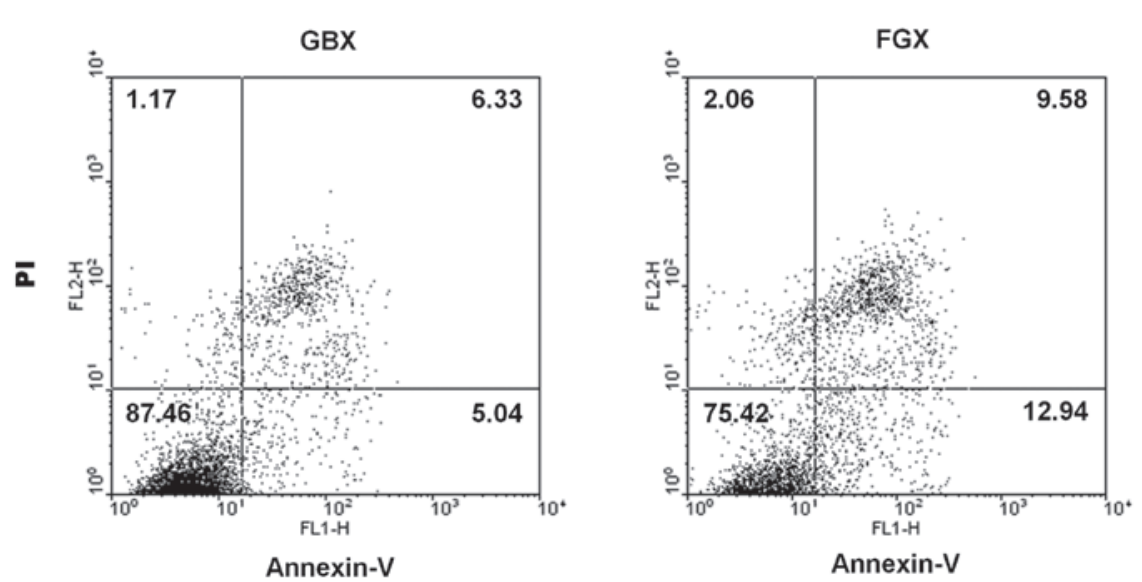

Figure 3. Effect of GBX or FGX on the induction of apoptosis of KATO3 cells. KATO3 cells were treated without or with GBX (125 $\mu \mathrm{g} / \mathrm{ml})$ and FGX $(125 \mu \mathrm{g} / \mathrm{ml})$ for $24 \mathrm{~h}$, respectively. The cells were double-stained with Annexin-V and PI, and then analyzed by flow cytometry. The numbers in the quadrants of each plot indicate the percentage of Annexin-positive (apoptotic) cells. Data are representative of three independent experiments. GBX, ginseng butanol extract; FGX, fortified ginseng extract. PI, propidium iodide.

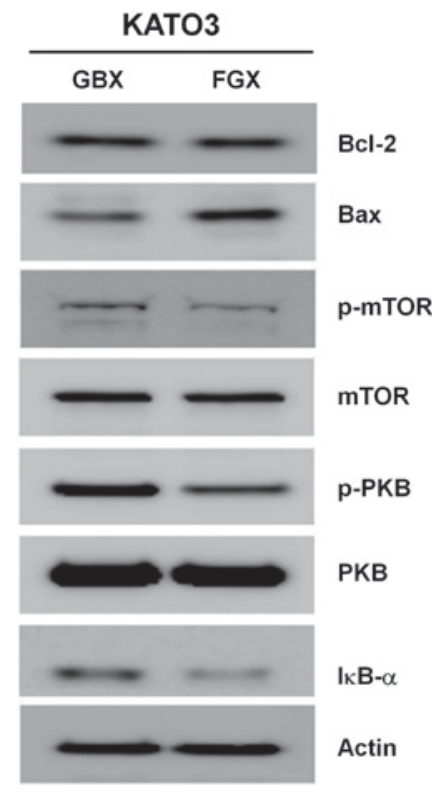

Figure 4. Effect of GBX or FGX on the expression and activity of growth-and/or signal-associated proteins in KATO3 cells. KATO3 cells were treated without or with GBX $(125 \mu \mathrm{g} / \mathrm{ml})$ and FGX $(125 \mu \mathrm{g} / \mathrm{ml})$ for $24 \mathrm{~h}$, respectively. Whole cell lysates were prepared and analyzed by western blotting using the respective antibodies. The expression levels of actin protein were used as a protein loading control. Each image is a representative of three independent experiments. Bcl-2, B-cell lymphoma 2; Bax, Bcl-2-associated $\mathrm{X}$ protein; $\mathrm{p}-\mathrm{mTOR}$, phosphorylated mammalian target of rapamycin; $\mathrm{PKB}$, protein kinase $B$; Iк $\mathrm{B} \alpha$, nuclear factor of kappa light polypeptide gene enhancer in B-cells inhibitor $\alpha$; GBX, ginseng butanol extract; FGX, fortified ginseng extract.

stages of apoptosis, and $\sim 9.58$ and $6.33 \%$ of cells in late apoptosis, respectively. These results confirmed the FGX-induced apoptosis of KATO3 cells.

FGX treatment leads to upregulation of Bax, dephosphorylation of $m T O R$ and $P K B$ and downregulation of $I \kappa B \alpha$ in KATO3 cells. Cell survival and/or apoptosis are largely affected by the expression and/or activity of a variety of proteins, including $\mathrm{Bcl}-2$ family proteins, $\mathrm{PKB}$ and mTOR. Therefore, the effects of FGX and GBX on the expression and/or activity (phosphorylation) of those proteins in KATO3 cells were examined by western blot analysis. As revealed in Fig. 4, compared with the effects of GBX (125 $\mu \mathrm{g} / \mathrm{ml}, 24 \mathrm{~h})$, treatment with FGX $(125 \mu \mathrm{g} / \mathrm{ml}, 24 \mathrm{~h})$ resulted in an increased expression of Bax, but did not affect Bcl-2 in KATO3 cells. Furthermore, compared with GBX, FGX further led to a decrease in the phosphorylated forms of PKB and mTOR in KATO3 cells, without affecting their total protein expression. There is further evidence that suggests the involvement of $\mathrm{NF}-\kappa \mathrm{B}$, a transcription factor, in cell survival and/or apoptosis. Activation of $\mathrm{NF}-\kappa \mathrm{B}$ is strongly correlated to the proteolysis of $\mathrm{I} \kappa \mathrm{B} \alpha$, a cytosolic NF- $\mathrm{B}$ inhibitory protein. This led us promptly to investigate the effect of FGX and GBX on the expression levels of $\mathrm{I} \kappa \mathrm{B} \alpha$ in KATO3 cells. Notably, there were lower expression levels of I $\mathrm{B} \alpha$ in FGX-treated KATO3 cells than in the GBX-treated cells.

\section{Discussion}

The pharmacological actions of the majority of ginseng preparations are attributed to ginsenosides (16). It is suggested that manufacturing methods, including steaming or heating, may enhance the anti-cancer activity of ginseng and/or ginsenosides over that of the original ginseng powder (17). In the present study, the effects of an enzymatically FGX and GBX, original ginseng extract on KATO3 human gastric cancer cells were compared. It was demonstrated that FGX had stronger anti-proliferative and pro-apoptotic effects on KATO3 cells than GBX. These data suggested that the FGX's anti-proliferative and pro-apoptotic effects on KATO3 cells appeared to be mediated via modulation of the expression and/or activities of $\mathrm{Bax}, \mathrm{I} \kappa \mathrm{B} \alpha / \mathrm{NF}-\kappa \mathrm{B}, \mathrm{mTOR}$ and PKB signaling agents.

Previously, it has been demonstrated that $\operatorname{Rg} 3$ and $\mathrm{Rh} 2$ block cell cycle progression of cancer cells (18-20) and compound $\mathrm{K}$ leads to cell cycle arrest in leukemia cells (21). A previous study by our group, also revealed that GBX, which contains high 
quantities of Rb1, inhibits growth and induces apoptosis of A549 human lung cancer cells (9), implying a positive role of Rb1 on the GBX-induced anti-growth and pro-apoptotic effects. In the present study, using HPLC-UV analysis, it was demonstrated that five ginsenosides, including Rh1, Rb1, Rg3(R), compound $\mathrm{K}$ and Rh2, were enriched in FGX compared with the ginsenoside profile in GBX. Importantly, the present study demonstrated lower numbers of KATO3 cells and more Annexin-V-stained populations following treatment with FGX as compared with the GBX-treated cells, suggesting that FGX had more potent anti-growth and pro-apoptotic activity in KATO3 cells than the original ginseng extract. These results may therefore indicate that the enzymatic method used in the present study may be useful for the production of a preparation of ginseng extract containing more ginsenosides with anti-cancer activity.

The induction of apoptosis is mediated through two main pathways; the intrinsic (mitochondrial) and the extrinsic (death receptor-mediated) pathways. Central to both apoptosis pathways are the caspases, a group of essential proteases required for the execution of programmed cell death by apoptotic stimuli $(22,23)$. Previously, it has been demonstrated that ginseng root extract induced mitochondrial-dependent apoptotic pathways in tumor cells (24), as deduced from the evidence that a caspase- 3 inhibitor was able to partially block the activation of caspase- 3 and the subsequent apoptotic cell death caused by ginseng root extract. Abundant data have suggested that the mitochondrial-dependent apoptotic pathways are largely affected by the expression and/or activity of several mitochondrial proteins, including Bcl-2 and Bax. Bcl-2 is an anti-apoptotic protein and participates in apoptosis initiation and/or caspase activation by regulating mitochondrial membrane integrity (25), while Bax is a pro-apoptotic protein and has a central role in cancer cell death by regulating mitochondrial outer-membrane permeabilization (26). Therefore, the ratio of $\mathrm{Bax} / \mathrm{Bcl}-2$ expression in the mitochondria is a key factor in the regulation of apoptosis induction (27). In the present study, it was demonstrated that treatment with FGX leads to upregulation of Bax protein, but does not affect the expression levels of the Bcl-2 protein. Therefore, it was hypothesized that FGX-induced upregulation of Bax may increase mitochondrial outer-membrane permeabilization and thereby facilitate the mitochondrial-mediated apoptotic pathways in KATO3 cells.

It has been reported that mTOR integrates multiple signals reflecting the availability of growth factors, nutrients or energy to promote cellular growth and/or to repress it (28). PKB is a well-established downstream effector of mTOR and, as a protein kinase, has been demonstrated to be involved in cell survival and/or apoptosis (29). Notably, in the present study, it was demonstrated that while there were substantial levels of phosphorylated mTOR and PKB in GBX-treated KATO3 cells, there was a marked diminishment of mTOR and PKB phosphorylation in the FGX-treated cells. It was thus hypothesized that FGX may interfere with the mTOR/PKB signals in KATO3 cells, which may contribute to FGX-induced reduction of cell survival and/or apoptosis of KATO3 cells.

Previously, it has been demonstrated that NF- $\kappa \mathrm{B}$ has dual functions, anti-apoptotic and pro-apoptotic, in cell survival responses (30), and its anti-apoptotic and/or pro-apoptotic function is largely dependent on the nature of the apoptotic stimuli and/or the type of cell (31). It is documented that activation (nuclear localization) of $\mathrm{NF}-\kappa \mathrm{B}$ is well associated with phosphorylation, ubiquitination and subsequent proteolytic degradation of $\mathrm{I} \kappa \mathrm{B} \alpha$, a $\mathrm{NF}-\kappa \mathrm{B}$ inhibitory protein (32). With this in mind, it is important to note the evidence of several earlier studies reporting that ginsenosides, including $\mathrm{Rh} 2, \mathrm{Rg} 3$ and compound $\mathrm{K}$, inhibited nuclear translocation of $\mathrm{NF}-\kappa \mathrm{B}$ (33-35), which suggested that these ginsenosides are able to inhibit $\mathrm{NF}-\kappa \mathrm{B}$. In the present study, however, a reduction in the expression levels of $\mathrm{I} \kappa \mathrm{B} \alpha$ protein in FGX-treated KATO3 cells as compared with the GBX-treated cells (Fig. 4) was demonstrated, which may imply FGX-induced proteolytic downregulation of $\mathrm{I} \kappa \mathrm{B} \alpha$ and thereby activation of $\mathrm{NF}-\kappa \mathrm{B}$. At present, the role of $\mathrm{I} \kappa \mathrm{B} \alpha$ downregulation (NF- $\kappa \mathrm{B}$ activation) in FGX-induced apoptosis and/or reduction of survival of KATO3 cells, however, remains elusive. Numerous studies have demonstrated that $\mathrm{NF}-\kappa \mathrm{B}$ may exert its pro-apoptotic activity through transcriptional induction of a number of pro-apoptotic genes, including death receptors, Fas ligand or Bcl-2 extra small, which, respectively, have NF- $\kappa \mathrm{B}$ cis-acting elements in their promoter regions (36-38). To elucidate the role of $\mathrm{NF}-\kappa \mathrm{B} / \mathrm{I} \kappa \mathrm{B} \alpha$ signals in FGX-induced apoptosis and/or the reduction of survival of KATO3 cells, further studies are required to investigate whether ectopic overexpression and/or small interfering RNA-mediated knockdown of NF- $\kappa \mathrm{B}$ affects the FGX's anti-proliferative and/or pro-apoptotic effects on KATO3 cells, and also which pro-apoptotic genes are differentially regulated under these experimental conditions.

In conclusion, the present study demonstrated for the first time, to the best of our knowledge, that FGX had stronger anti-growth and pro-apoptotic effects on KATO3 cells than GBX, and the FGX's anti-growth and pro-apoptotic effects appear to be associated with the upregulation of $\mathrm{Bax}, \mathrm{I} \kappa \mathrm{B} \alpha$ proteolysis-mediated activation of $\mathrm{NF}-\kappa \mathrm{B}$, and the inhibition of mTOR and PKB signals. It is proposed that FGX may be used, as a single and/or combined treatment, with known anti-cancer drugs, against human gastric cancer cells.

\section{Acknowledgements}

This study was supported by the National Research Foundation of Korea Grant funded by the Korean Government (MEST) (2013, University-Institute cooperation program) and was also in part supported by a Korea Basic Science Institute grant (D33403) to J.S. Choi.

\section{References}

1. Asafu-Adjaye EB and Wong SK: Determination of ginsenosides (ginseng saponins) in dry root powder from Panax ginseng, Panax quinquefolius, and selected commercial products by liquid chromatography: interlaboratory study. J AOAC Int 86: 1112-1123, 2003.

2. Yun TK, Lee YS, Lee YH, Kim SI and Yun HY: Anticarcinogenic effect of Panax ginseng C.A. Meyer and identification of active compounds. J Kor Med Sci 16: S6-S18, 2001.

3. He BC, Gao JL, Luo X, Luo J, Shen J, Wang L, Zhou Q, Wang YT, Luu HH, Haydon RC, Wang CZ, Du W, Yuan CS, He TC and Zhang BQ: Ginsenoside Rg3 inhibits colorectal tumor growth through the down-regulation of Wnt/-catenin signaling. Int J Oncol 38: 437-445, 2011.

4. Kim do Y, Park MW, Yuan HD, Lee HJ, Kim SH and Chung SH: Compound $\mathrm{K}$ induces apoptosis via CAMK-IV/AMPK pathway in HT-29 colon cancer cells. J Agric Food Chem 57: 10573-10578, 2009. 
5. Park HM, Kim SJ, Kim JS and Kang HS: Reactive oxygen mediated ginsenoside Rg3- and Rh2-induced apoptosis in hepatoma cells through mitochondrial signaling pathways. Food Chem Toxicol 50: 2736-2741, 2012.

6. Mai TT, Moon J, Song Y, Viet PQ, Phuc PV, Lee JM, Yi TH, Cho $\mathrm{M}$ and Cho SK: Ginsenoside F2 induces apoptosis accompanied by protective autophagy in breast cancer stem cells. Cancer Lett 321: 144-153, 2012.

7. Shibata S: Chemistry and cancer preventing activities of ginseng saponins and some related triterpenoid compounds. J Kor Med 16: S28-S37, 2001.

8. Hwang JW, Oh JH, Yoo HS, Lee YW, Cho CK, Kwon KR, Yoon JH, Park J, Her S, Lee ZW, Jang IS and Choi JS: Mountain ginseng extract exhibits anti-lung cancecr activity by inhibiting the nuclear translocation of NF-кB. Am J Chin Med 40: 187-202, 2012.

9. Lee DG, Jang SI, Hwang JW, Yang KE, Yoon SJ, Lee ZW, An HJ, Jang IS, Choi, JS and Yoo HS: Anti-proliferative effects of ginsenosides extracted from mountain ginseng on lung cancer. Chin J Integ Med (In press).

10. Suh SO, Kroh M, Kim NR, Joh YG and Cho MY: Effects of red ginseng upon postoperative immunity and survival in patients with stage III gastric cancer. Am J Chin 30: 483-494, 2002.

11. Crew KD and Neugut AI: Epidemiology of gastric cancer. World J Gastroenterol 12: 354-362, 2006.

12. Meyer HJ and Wilke $\mathrm{H}$ : Treatment strategies in gastric cancer Dtsch Arztebl Int 108: 698-706, 2011.

13. Jain VK, Cunningham D and Chau I: Preoperative and postoperative chemotherapy for gastric cancer. Surg Oncol Clin N Am 21: 99-112, 2012.

14. Scartozzi M, Galizia E, Verdecchia L, Berardi R, Antognoli S, Chiorrini S and Cascinu S: Chemotherapy for advanced gastric cancer: across the years for a standard of care. Expert Op Pharmacother 8: 797-908, 2007.

15. Al-Batran SE, Ducreux M and Ohtsu A: mTOR as a therapeutic target in patients with gastric cancer. Int J Cancer 130 491-496, 2012

16. Attele AS, Wu JA and Yuan CS: Ginseng pharmacology: multiple consitituents and multiple actions. Biochem Pharmacol 58: 1685-1693, 1999.

17. Christensen LP: Ginsenosides chemistry, biosynthesis, analysis, and potential health effects. Adv Food Nutr Res 55: 1-99, 2009.

18. Kim SM, Lee SY, Cho JS, Son SM, Choi SS, Yun YP, Yoo HS, Yoon do Y, Oh KW, Han SB and Hong JT: Combination of ginsenoside $\operatorname{Rg} 3$ with docetaxel enhances the susceptibility of prostate cancer cells via inhibition of NF-kappa B. Eur J Pharmacol 631: 1-9, 2010.

19. Lee KY, Park JA, Chung E, Lee YH, Kim SI and Lee SK: Ginsenoside-Rh2 blocks the cell cycle of SK-HEP-1 cells at the G1/S boundary by selectively inducing the protein expression of p27 (kip1). Cancer Lett 110: 193-200, 1996.

20. Park JA, Lee KY, Oh YJ, Kim KW and Lee SK: Activation of caspase-3 protease via a Bcl-2 insensitive pathway during the process of ginsenoside Rh2-induced apoptosis. Cancer Lett 121: 73-81, 1997.

21. Kang KA, Kim YW, Kim SU, Chae S, Koh YS, Kim HS, Choo MK, Kim DH and Hyun JW: G1 phase arrest of the cell cycle by a ginseng metabolite, compound $\mathrm{K}$, in U937 human monocytic leukemia cells. Arch Pharm Res 28: 685-690, 2005.
22. Slee EA, Adrain C and Martin SJ: Serial killers: ordering caspase activation events in apoptosis. Cell Death Differ 6: 1067-1074, 1999.

23. Cohen GM: Caspases: the executioners of apoptosis. Biochem J 326: 1-16, 1997.

24. Mohamad N, Gutiérrez A, Núñez M, Cocca C, Martín G, Cricco G, Medina V, Rivera E and Bergoc R: Mitochondrial apoptotic pathways. Biocell 29: 149-161, 2005.

25. Adams JM and Cory S: The Bcl-2 protein family: arbiters of cell survival. Science 281: 1322-1326, 1998

26. Jourdain A and Martinou JC: Mitochondrial outer-membrane permeabilization and remodelling in apoptosis. Int J Biochem Cell Biol 41: 1884-1889, 2009.

27. Zinkel S, Gross A and Yang E: BCL2 family in DNA damage and cell cycle control. Cell Death Differ 13: 1351-1359, 2006.

28. Fingar DC and Blenis J: Target of rapamycin (TOR): an integrator of nutrient and growth factor signals and coordinator of cell growth and cell progression. Oncogene 23: 3151-3171, 2004.

29. Sarbassov DD, Guertin DA, Ali SM and Sabatini DM: Phosphorylation and regulation of Akt/PKB by the rictor-mTOR complex. Science 307: 1098-1101, 2005.

30. Graham B and Gibson SB: The two faces of NFkB in cell survival responses. Cell Cycle 4: 1342-1345, 2005.

31. Kaltschmidt B, Kaltschmidt C, Hofmann TG, Hehner SP, Dröge W and Schmitz ML: The pro- or anti-apoptotic function of NF-kappaB is determined by the nature of the apoptotic stimulus. Eur J Biochem 267: 3828-3835, 2000.

32. Ghosh S and Baltimore D: Activation in vitro of NF-kappa B by phosphorylation of its inhibitor I kappa B. Nature 344: 678-682, 1990 .

33. Bi WY, Fu BD, Shen HQ, Wei Q, Zhang C, Song Z, Qin QQ, Li HP, Lv S, Wu SC, Yi PF and Wei XB: Sulfated derivative of 20(S)-ginsenoside Rh2 inhibits inflammatory cytokines through MAPKs and NF-kappa B pathways in LPS-induced RAW264.7 macrophages. Inflammation 35: 1659-1668, 2012

34. Kim ND, Kim EM, Kang KW, Cho MK, Choi SY and Kim SG: Ginsenoside Rg3 inhibits phenylephrine-induced vascular contraction through induction of nitric oxide synthase. Br J Pharmacol 40: 661-670, 2003.

35. Ming Y, Chen Z, Chen L, Lin D, Tong Q, Zheng Z and Song G: Ginsenoside compound $\mathrm{K}$ attenuates metastatic growth of hepatocellular carcinoma, which is associated with the translocation of nuclear factor-KB p65 and reduction of matrix metalloproteinase-2/9. Planta Med 77: 428-433, 2011.

36. Matsui K, Fine A, Zhu B, Marshak-Rothstein A and Ju ST: Identification of two NF-kappa B sites in mouse CD95 ligand (Fas ligand) promoter: functional analysis in T cell hybridoma. J Immunol 161: 3469-3473, 1998

37. Ravi R, Bedi GC, Engstrom LW, Zeng Q, Mookerjee B, Gélinas C, Fuchs EJ and Bedi A: Regulation of death receptor expression and TRAIL/Apo2L-induced apoptosis by NF- $\kappa$ B. Nat Cell Biol 3: 409-416, 2001.

38. Zheng Y, Ouaaz F, Bruzzo P, Singh V, Gerondakis S and Beg AA: NF- $\kappa$ B RelA (p65) is essential for TNF-a-induced fas expression but dispensable for both TCR-induced expression and activation-induced cell death. J Immunol 166: 4949-4957, 2001. 